\title{
Gestión educativa ambiental y responsabilidad social universitaria en la UNAC 2021
}

\author{
Mg. Lic. Cristhians Omar De La Torre Collao \\ cdelatorrec@ucvvirtual.edu.pe \\ https://orcid.org/0000-0001-6313-2295
}

Dr. Lic. Luis Alberto De La Torre Collao

delatorreluis@yahoo.es https://orcid.org/0000-0001-7473-3264

\section{Lic. Gustavo Alonso De La Torre Bocanegra delatorrebgustavo@gmail.com https://orcid.org/0000-0003-0586-6834}

Escuela de Pos Grado Universidad Cesar Vallejo Lima Perú

\section{RESUMEN}

El presente estudio surgió como parte de la indagación de los temas medio ambientales que afecta al desarrollo educativo en el nivel formativo profesional en la Región Callao. Tuvo como objetivo determinar la relación existente entre la Gestión de la Educación Ambiental y La responsabilidad Social Universitaria (RSU) tomando aspectos relevantes de la percepción que se tiene en los educadores universitarios de la Universidad Nacional del Callao en la Provincia Constitucional del primer puerto del país. Abordándose un enfoque cuantitativo y un diseño no experimental, transversal, tipo aplicado y nivel correlacional, se diseñó una encuesta estructurada conformada por 32 ítems a una muestra conformada por 61 docentes universitarios de la facultad de Ciencias Administrativas de los cuales se obtuvo una confiabilidad con coeficiente de Cronbach de 0.943 y 0.936 para las variables desarrolladas, obteniéndose un resultado mediante Rho de Spearman de 0,430 percibiéndose una correlación estadísticamente moderada con un nivel de significancia bilateral menor de 0,05 que permitió aceptar la hipótesis en análisis.

Palabras Clave: gestión educativa ambiental; responsabilidad social universitaria 


\title{
Environmental educational management and university social responsibility at UNAC 2021
}

\begin{abstract}
This study arose as part of the investigation of environmental issues that affect educational development in the professional training stage in the Callao Region. Its objective was to determine the existing relationship between the Management of Environmental Education and University Social Responsibility (RSU), taking relevant aspects of the perception that is had in the university teachers of the National University of Callao in the Constitutional Province of the first port of the country. Addressing a quantitative approach and a non-experimental, cross-sectional design, applied type and correlational level, a structured survey was designed consisting of 32 items to a sample made up of 61 university professors from the Faculty of Administrative Sciences of which a reliability with coefficient was obtained. Cronbach's of 0.943 and 0.936 for the developed variables, obtaining a result by Spearman's Rho of 0.430 , perceiving a statistically moderate correlation with a bilateral significance level of less than 0.05 that allowed accepting the hypothesis in analysis.
\end{abstract}

Keywords: environmental educational management; university social responsibility

Artículo recibido: 30 noviembre. 2021 Aceptado para publicación: 29 diciembre 2021 Correspondencia: cdelatorrec@ucvvirtual.edu.pe Conflictos de Interés: Ninguna que declarar 


\section{INTRODUCCIÓN}

La presente investigación describió las implicancias de la educación ambiental y las consecuencias de las incidencias ambientales que se ha hecho cada vez más diversa, no solo abarcando a seres vivos sino también a las consecuencias producidas por la extenuación del recubrimiento de ozono y por ende el calentamiento global, la contaminación de los mares, los ríos, el descongelamiento de la antártica, en ese sentido, la aparición de cuantiosas teorías en Educación Ambiental (EA) tiene una procedencia de la aplicación diversos paradigmas propios en este campo desde hace más de 40 años, especificando el escenario de las décadas de los 70 y 80 , que provienen de la organización de los instrumentos utilizados como el estudio al funcionamiento de la indagación de iniciativas pedagógicas en ese ambiente, y no como un límite que clasifique la acción ambiental en categorías rígidas con el fin de cambiar la realidad (Sauvé, 2005). En consecuencia, se puede detallar varias vertientes con gran tradición en esta importante actividad como son moral-ética, resolutiva, naturalista, conservacionista, sistémica, humanista y científica.

Por otra parte al comprender la EA como una actividad que logre impacto no solo en el entorno medioambiental que sin duda es imprescindible para sostener los diversos ecosistemas; y más aún sin omitir la participación de los ciudadanos como principal ejecutores de las acciones percibidas, los valores inculcados (González, 2017), (Raudah, Hidir, Nor, y Erliani, 2021) menciona que la cultura del entorno y la generación de nuevos conocimientos a través de la heurística tiene la capacidad para realizar de modo inmediato innovaciones positivas para sí mismo y para sus propósitos. Se han identificado algunas dimensiones para explicar el entorno en el que se ejecuta la educación ambiental partiendo de la premisa y la importancia al cambio del entorno, en ese sentido la dimensión del conocimiento que representa lo que sabemos y comprendemos del mundo real, la dimensión de las habilidades, que se basa en cómo usamos lo que sabemos, la dimensión de las actitudes que interpreta el cómo nos comportamos y comprometemos en el mundo y finalmente, el de la meta de aprendizaje que describe cómo nos reflejamos y adaptamos a los entornos cambiantes (Fadel, Bialik, y Trilling, 2015).

Por ende, (Olaguez, Peña, y Espino, 2017) refiere que es requerido la aplicación de una educación ambiental que se considera un modelo teórico, metodológico y práctico que 
propaga el sistema educativo tradicional y alcanza la noción de medio ambiente y de desarrollo. Mientras que (Ikram, Zhou, Shah, y Liu, 2019) alude que es un proceso educativo para cada sujeto, las familias, las comunidades, la sociedad y el Estado, para tomar decisiones y actuar en pro del desarrollo sostenible mundial.

La finalidad de la formación estructurada en la educación ambiental es la adquisición de habilidades para el manejo de los métodos, técnicas o herramientas propias de su profesión, necesarias para poder intervenir en la detección, prevención y/o corrección de los problemas ambientales (Callejas, Sáenz, Plata, Holguín, y Mora, 2018).

En ese contexto, en el marco de los componentes epistemológicos, axiológicos y metodológicos con los que se fundamenta la argumentación de la existencia de la ciencia sustentada y la cosmovisión con la que se perciben en un paradigma positivista con la que se enmarcó la estrategia a seguir en la investigación, (Arza, 2017) reconoce el mecanismo que obra en el adoctrinamiento no obligatorio (siempre sobre los datos tomados en Piamonte): en general, el padre es el principal fuente de ingreso de la descendencia y a los 65 años se jubila (con lo cual, los ingresos de la familia se reducen notablemente), por lo que (Mertens, 2017) indica que la educación superior es una versión considerable para la cual el mercado bancario no proporciona buenos préstamos; ante la futura debilidad económica, es de esperar que se estimule emprendimientos sostenibles en los hijos a una temprana entrada al mercado laboral. Así se explica por qué distintos progenitores y descendientes, en situaciones diversas, adoptan decisiones similares, lo cual genera un fenómeno social en la explicación al desarrollo y problemática de la gestión de la educación ambiental como elemento primordial dentro de los aspectos del valor público en los centros de enseñanza superior universitaria en la Provincia Constitucional del Callao como parte del aporte y de criterios de comparación en un escenario fundamentado en la Política Nacional de Educación Ambiental (PNEA), aprobada mediante D.S. 017-2012-ED, establece los objetivos, lineamientos de política y resultados esperados en la formación y fortalecimiento de una ciudadanía responsable con el ambiente y su entorno, en el marco del desarrollo sostenible a nivel nacional (Ministerio del Ambiente, 2012).

En el escenario actual en el que la colectividad en su totalidad y en singular, la comunidad académica, científica y empresarial requiere de hechos que evidencia la preservación de los ecosistemas y conservación de los entornos culturales mediante los 
diversos agentes y stakholders en el ámbito educativo. En ese contexto, se considera a la RSU como un articulador que genera voluntad y optimización de los diferentes procesos en el ámbito educativo superior mediante los diversos estándares basaos en la estrategia de gestión, el proceso articulador y una postura política sostenida por una interacción participativa e innovadora en los entornos sociales para el perfeccionamiento del confort vital de las personas (Laurencio y Farfán Pacheco, 2016). En ese constructo de ideas la principal interrogante que se abordó fue ¿De qué manera está relacionada la gestión de la educación ambiental y la responsabilidad social en la educación universitaria de la Provincial Constitucional del Callao? El objetivo de esta pesquisa fue precisar la conexión entre la gestión de la educación ambiental y la responsabilidad social en la actividad superior universitaria.

(Olaguez et al., 2017), consideraron que es indispensable adecuar los planes curriculares de la Universidad teniendo como una experiencia curricular los temas de carácter ambiental para tomando como eje la disminución de las barreras como desigualdades económicas, la contaminación, el desequilibrio de los ecosistemas que genera mayores índices de pobreza local. (Álvarez y Hernández, 2021), Han demostrado que los estudiantes de educación secundaria valoran las pruebas sostenidas para el conocimiento y participación en actividades ambientales, mediante aplicativos virtuales utilizando los Smartphone, sin embargo, se apreció que esta reduce a medida que aumenta los años de los jóvenes por lo que es necesario sensibilizar al colectivo para incrementar el interés por el cuidado del planeta empleando instrumentos tecnológicos. (Callejas et al., 2018), actualizaron la percepción que se tiene en las entidades educativas de nivel superior como parte de institucionalizar la actividad ambiental como parte del compromiso a través de la docencia, investigación apoyándose de las políticas institucionales y gubernamentales en favor de la clase académica como principal soporte al desarrollo de nuevos conocimientos en este campo por lo que es indispensable aplicar estrategias sostenibles de autoevaluación regulatoria para promover la institucionalidad en el país. (E. Mendoza, Boza, y Escobar, 2019), Evidenció que existe considerables indicios de ignorancia sobre la cultura ambiental y sobre la cosmovisión que aborda la temática como una fuente de mejora significativa en las entidades educativas a nivel superior, reflejándose con mayor énfasis en los estratos sociales bajos producto de las inacciones administrativas y una adecuada visión 
que articule los actores con la comunidad académica. (E. Mendoza et al., 2019) concordaron en su pesquisa que existen indicios razonables y metodológicos que demostraron que la población estudiantil atribuye eventualmente los valores educativo ambientales en su común devenir diario. No obstante, hay un alto porcentaje de alumnos que una reducida praxis en esta materia ocasiona una degradación de los ecosistemas. (Ordóñez, Montes, y Garzón, 2018), consideraron que la EA es un campo que se ha extendido a través de américa Latina como resultado de diversas gestiones gubernamentales y grupos de interés en lo académico y social, sin embargo, es necesario establecer mecanismos que optimicen la apertura de centros para la reducción de riesgos, así como también incrementar las opciones de respuesta inmediata ante acontecimientos que pongan en grave riesgo la salud e integridad física de la sociedad. (Brito y Castillo, 2018), afirmaron que la EA es una actividad imprescindible desde los primeros años de vida en tanto la sensibilización a los infantes en adiestramiento escolar busca crear nuevas actitudes y compromiso con manejo responsable del desecho de residuos sólidos, así como la preservación del medio ambiente.

\section{ESTRATEGIAS METODOLÓGICAS O MATERIALES Y MÉTODOS}

El presente estudio se sostuvo bajo un enfoque cuantitativo y un diseño no experimental a razón que no se maniobró las variables de investigación el cual se tomó en un determinado momento bajo el tipo transaccional, correlacional y explicativo. Se aplicó una técnica de recolección de datos basado en una encuesta y un instrumento de tipo cuestionario compuesto por 12 preguntas para medir la Gestión Educativa Ambiental y 20 ítems para el tema de Responsabilidad Social Universitaria a una muestra conformada por 61 Docentes contratados y nombrados de la Facultad de Ciencias Administrativas de la UNAC. En consecuencia, utilizando el análisis matemático y estadístico bajo la aplicación del software SPSS versión 25 para contrastar y entender objetivamente la hipótesis planteada que centró al investigador en un dilema optimista sobre las variables que formaron parte del estudio, se sometió ambos instrumentos al procedimiento de validación a criterio de expertos y confiabilidad bajo el coeficiente de alfa de Cronbach del cual se obtuvo un valor de 0.879 y 0.912 . La medida de la confiabilidad interna de los instrumentos se establece mediante el alfa de Cronbach, que asume que los ítems (medidos en escala tipo Likert) miden un mismo constructo y que están altamente correlacionados (Welch y Comer, 1989). Cuanto más cerca se encuentre 
el valor del alfa a 1 mayor es la consistencia de los ítems analizados demostrando que mientras tantas veces se aplique el instrumento de estudio, los resultados analizados serán similares.

\section{RESULTADOS Y DISCUSIÓN}

\section{Tabla 1}

Prueba de Normalidad de las Variables

\begin{tabular}{cllc}
\multirow{2}{*}{ Variables } & \multicolumn{3}{l}{ Kolmogorov-Smirnov $^{\mathbf{a}}$} \\
\cline { 2 - 4 } & Estadístico & gl & Sig. \\
\hline Gestión de la educación ambiental & 0.179 & 61 & 0.000 \\
Responsabilidad social universitaria & 0.115 & 61 & 0.042 \\
\hline
\end{tabular}

Nota. ${ }^{\text {a }}$ Corrección de significación de Lilliefors

Como se puede apreciar, ambas variables no poseen una distribución estadísticamente normal en ese contexto, se aplicó la prueba se Kolmogorov-Smirnov lo cual permite aplicar un análisis de correlación con Rho de Spearman.

\section{Tabla 2}

Descripción de las variables Gestión de la educación ambiental y Responsabilidad social universitaria con sus respectivas dimensiones

\begin{tabular}{|c|c|c|c|}
\hline Variable & Dimensión & Nivel & n (\%) \\
\hline \multirow{9}{*}{$\begin{array}{l}\text { Gestión de la educación } \\
\text { ambiental }\end{array}$} & \multirow{3}{*}{ Conocimiento } & Bajo & $10(16.4)$ \\
\hline & & Medio & $39(63.9)$ \\
\hline & & Alto & $12(19.7)$ \\
\hline & \multirow{3}{*}{ Actuación } & Bajo & 12 (19.7) \\
\hline & & Medio & $34(55.7)$ \\
\hline & & Alto & $15(24.6)$ \\
\hline & \multirow{3}{*}{ Participación } & Bajo & $22(36.1)$ \\
\hline & & Medio & $25(41.0)$ \\
\hline & & Alto & $14(23.0)$ \\
\hline \multirow{11}{*}{$\begin{array}{l}\text { Responsabilidad social } \\
\text { universitaria }\end{array}$} & \multirow{3}{*}{$\begin{array}{l}\text { Transformación } \\
\text { personal }\end{array}$} & Bajo & $12(19.7)$ \\
\hline & & Medio & $42(68.9)$ \\
\hline & & Alto & $7(11.5)$ \\
\hline & \multirow{3}{*}{$\begin{array}{l}\text { Transformación de } \\
\text { relaciones }\end{array}$} & Bajo & $8(13.1)$ \\
\hline & & Medio & $35(57.4)$ \\
\hline & & Alto & $18(29.5)$ \\
\hline & \multirow[t]{2}{*}{ Patrones culturales } & Bajo & $11(18.0)$ \\
\hline & & Medio & $50(82.0)$ \\
\hline & \multirow{3}{*}{$\begin{array}{l}\text { Transformación } \\
\text { estructural }\end{array}$} & Bajo & $10(16.4)$ \\
\hline & & Medio & $35(57.4)$ \\
\hline & & Alto & $16(26.2)$ \\
\hline
\end{tabular}


Del análisis efectuado se puede describir que la articulación de la gestión de la educación ambiental tiene un nivel medio de $63.9 \%$ para la dimensión conocimiento, $55.7 \%$ para la dimensión actuación y finalmente un $41 \%$ para participación. Del mismo modo, la RSU en su dimensión transformación personal tiene un 68..9\% seguido de transformación de relaciones con un57.4\% así mismo, los patrones culturales con un $82 \%$ y por ultimo la transformación estructural con un valor de $57.4 \%$

\section{Tabla 3}

Correlación de variable Gestión de la educación ambiental y Responsabilidad social universitaria

\begin{tabular}{lcc}
\hline \multicolumn{1}{c}{ Rho de Spearman } & Responsabilidad social universitaria \\
\hline $\begin{array}{l}\text { Coeficiente de Gestión de la educación } \\
\text { correlación ambiental } \\
\text { Sig. (bilateral) }\end{array}$ &, $430^{* *}$ \\
\cline { 2 - 3 } & $\mathrm{N}$ & 0.001 \\
\hline
\end{tabular}

Nota. $* *$ La correlación es significativa en el nivel 0,01 (bilateral)

De acuerdo a las pesquisas del cual se analizó con un nivel de significancia de 0,01 la misma que se ubicó dentro del valor permitido $(\mathrm{p}<$ de 0,05$)$ se admitió la hipótesis general. De la cual se encontró una relación lineal estadísticamente moderada $(r=$ 0,430) y directamente proporcional (con signo positivo) entre la Gestión de la Educación Ambiental y responsabilidad social Universitaria.

\section{Tabla 4}

Correlación entre las dimensiones de la variable Gestión de la educación ambiental con la Responsabilidad social universitaria

\begin{tabular}{|c|c|c|c|}
\hline Variable & Dimensión & Rho de Spearman & $\begin{array}{c}\text { Responsabilidad social } \\
\text { universitaria }\end{array}$ \\
\hline \multirow{6}{*}{$\begin{array}{l}\text { Gestión de la } \\
\text { educación } \\
\text { ambiental }\end{array}$} & \multirow[t]{2}{*}{ Conocimiento } & $\begin{array}{c}\text { Coeficiente } \\
\text { correlación }\end{array}$ &, $354^{* *}$ \\
\hline & & Sig. (bilateral) & 0.005 \\
\hline & \multirow[t]{2}{*}{ Actuación } & $\begin{array}{c}\text { Coeficiente } \\
\text { correlación }\end{array}$ & 0.143 \\
\hline & & Sig. (bilateral) & 0.271 \\
\hline & \multirow[t]{2}{*}{ Participación } & $\begin{array}{l}\text { Coeficiente de } \\
\text { correlación }\end{array}$ &, $597^{* *}$ \\
\hline & & Sig. (bilateral) & 0.000 \\
\hline
\end{tabular}


Según los datos procesados, se puede precisar que existe una correlación moderada entre el conocimiento y la RSU con un valor 0,354 . Por otra parte se pudo contrastar la aceptación de Ho en vista de que no existe relación entre la actuación y la RSU del cual se obtuvo un valor de 0.143 Finalmente se pudo evidenciar una correlación moderada entre la participación y la RSU con un valor de 0,597

Considerando el objetivo de investigación se aprecia que el valor del Rho Spearman resultante es de 0,430 apreciándose que la educación ambiental tiene una asociación positiva con la responsabilidad social universitaria revelando que al tomar en cuenta las políticas gubernamentales en pro de la mejora de la calidad de vida en la comunidad universitaria de la Provincia Constitucional de Callao es una vía de solución a considerar en la sensibilización de toda organización estudiantil a nivel superior. Lo sostenido tiene potencial relevancia con lo contrastado por (Olaguez et al., 2017), quienes sostuvieron que bajo el coeficiente de Kendall $(0,094381)$ y chi cuadrado de x2=65,12 y p valúe $(4,07)$ con lo que lo los estudiantes universitarios perciben que debe involucrarse la formación ambiental como una asignatura indispensable en la formación universitaria. En razón del conocimiento percibido por los encuestados como parte del primer objetivo específico de investigación. Se evidenció que el valor de 0,354 refleja una correlación estadísticamente moderada que pone en evidencia los esfuerzos que se debe aunar para desarrollar aspectos cognitivos como resultado de la difusión de RSU en el sistema universitario que se respalda en lo presentado por (Estrada, Huaypar, y Mamani, 2020) que concluyeron que la EA debe generar nuevos conocimientos a través de la guía docente previo potenciamiento de capacidades y habilidades metodológicas para lograr un impacto positivo en los proyectos de mejora continua en la clase estudiantil. Por otra parte, revisando el segundo objetivo de estudio se pudo determinar que no existe relación entre la actuación y la RSU por lo que se obtuvo el valor $\mathrm{P}$ 0,143 con una significancia bilateral 0,271 que pone en manifiesto que hay una carencia de elementos de gestión que midan los resultados de frecuencia activa en las actividades ambientales lo que se apoya en la investigación presentada por (H. Mendoza, Loayza, y González, 2019), que enfatizan su postura sobre la falta de incentivo en la promoción ambiental en las instituciones educativas superiores lo que ocasiona deserción de los actores que ejecutan y difunden los emprendimientos de cambio en el contexto medio ambiental. Finalmente se ha respecto al objetivo de participación se evidenció una 
correlación moderada cuyo valor se detalla en 0,597 aplicado mediante el coeficiente de Rho de Spearman con un nivel de significancia de 0,000 lo que lleva a la reflexión sobre todos las acciones que se deben potenciar con apoyo de los gobiernos Locales, Regionales, la sociedad en su conjunto, medios de comunicación entre otros para impulsar la participación activa que corrobora lo descrito por (Brito y Castillo, 2018), que abordan la problemática de falta de participación en los programas para formar un pensamiento crítico, prospectivo en los niños y así transmitir calidad de vida a las futuras generaciones.

\section{CONCLUSION O CONSIDERACONES FINALES}

El valor de Rho de Spearman resultante del estudio es de 0,430 visualizando que la gestión de la educación ambiental posee una relación moderada con la responsabilidad social universitaria.

Del Mismo modo aplicando el análisis previamente mencionado, el conocimiento refleja una relación estadísticamente moderada frente a la RSU revelando que posiblemente es necesario una mayor difusión al cuerpo docente universitario para sensibilizarlo y lograr replicar ante la comunidad estudiantil los beneficios de la cultura ambientalista.

Por otra parte, en el análisis aplicado pudo reflejar que la dimensión acción no tiene relación con la RSU en vista que se obtuvo un valor de 0,143 razón por la cual las autoridades académicas deben reformular los planes estratégicos a fin de contemplar en su visión, misión y objetivos organizacionales las acciones ambientales que logren articular esfuerzos conjuntos en la académica.

Finalmente se aplicando la inferencia estadística se puede concluir que la participación posee una relación moderada con la RSU que como resultado de la indagación procesamiento y análisis se obtuvo un valor de 0,597 en ese contexto, es necesario aplicar estrategias conjuntas con los grupos de interés para involucrar a todo el personal docente en la importancia de promover permanentemente el cuidado del medio ambiente en la Provincia Constitucional del Callao así como también articular a las instituciones educativas del nivel inicial primario y secundario como parte de la visión en materia ambiental del país al año 2050. 


\section{LISTA DE REFERENCIAS}

Álvarez, J., y Hernández, J. (2021). Itinerarios didácticos con smartphones para promover la educación ambiental y la competencia digital entre el alumnado de secundaria. Digital Education Review, (39), 319-335.

Arza, C. (2017). El diseño de los sistemas de pensiones y la igualdad de género ¿Qué dice la experiencia europea? Serie Mujer y Desarrollo, 142(2), 5-45.

Brito, M., y Castillo, L. (2018). Gestión ambiental comunitaria para las niñas y niños del consejo popular Carlos Manuel. Avances, 20(3), 298-307.

Callejas, M., Sáenz, O., Plata, A., Holguín, M., y Mora, W. (2018). El compromiso ambiental de instituciones de educación superior en Colombia. Praxis \& Saber, 9(21), 197-220.

Estrada, E., Huaypar, K., y Mamani, H. (2020). La educación ambiental y el manejo de residuos sólidos en una institución educativa de Madre de Dios, Perú. Ciencia Amazónica (Iquitos), 8(2), 239-252.

Fadel, C., Bialik, M., y Trilling, B. (2015). Four Dimansional Educatión: The Competencies Learners Need to Succeed (1. a ed.). United States of America: Center for Curriculum Redesign.

González, C. (2017). La educación ambiental ante el problema ético del desarrollo. Revista Electrónica Educare, 21(2), 1-19.

Ikram, M., Zhou, P., Shah, S. A. A., y Liu, G. Q. (2019). Do environmental management systems help improve corporate sustainable development? Evidence from manufacturing companies in Pakistan. Journal of Cleaner

Production, 226, 628-641.

Laurencio, A., y Farfán Pacheco, P. (2016). La innovación educativa en el ámbito de la responsabilidad social universitaria. Revista Cubana de Educación Superior, 35(2), 16-34.

Mendoza, E., Boza, J., y Escobar, H. (2019). Educación ambiental y la práctica de valores de los estudiantes universitarios. Revista Cognosis, 4(2), 25-40.

Mendoza, H., Loayza, M., y González, M. (2019). Análisis de la gestión ambiental como indicador de la responsabilidad social universitaria. Revista Universidad y Sociedad, 11(2), 37-41. 
Mertens, D. (2017). Borrowing for social security? Credit, asset-based welfare and the decline of the German savings regime. Journal of European Social Policy, 27(5), 474-490.

Ministerio del Ambiente. (2012). Decreto Supremo $N^{\circ}$ 017-2012-ED. Recuperado de https://www.minam.gob.pe/wp-content/uploads/2013/09/ds_017-2012-ed.pdf

Olaguez, J., Peña, E., y Espino, P. (2017). La gestión de la educación ambiental en las organizaciones desde la perspectiva de los estudiantes de la universidad politécnica del valle del évora. HOLOS, 8, 145.

Ordóñez, M., Montes, L. M., y Garzón, G. (2018). Importancia de la educación ambiental en la gestión del riesgo socio-natural en cinco países de América Latina y el Caribe. Revista Electrónica Educare, 22(1), 345-363.

Raudah, R., Hidir, A., Nor, M., y Erliani, S. (2021). Understanding Educational Management in The Context of Environmental Protection for Madrasah Application. Nazhruna: Jurnal Pendidikan Islam, 4(2), 419-433.

Sauvé, L. (2005). Una cartografía de corrientes en Educación Ambiental. En Educação ambiental-Pesquisa e desafios. (pp. 17-46). Porto alegre: Armed.

Welch, S., y Comer, J. (1989). Quantitative Methods for Public Administration: Techniques and Applications. California: Brooks/Cole Publishing Co. 